\title{
Incremental value of contrast myocardial perfusion to detect intermediate versus severe coronary artery stenosis during stress-echocardiography
}

\author{
Nicola Gaibazzi ${ }^{+1}$, Fausto Rigo ${ }^{\dagger 2}$, Angelo Squeri+1, Fabrizio Ugo ${ }^{\dagger 1}$ and Claudio Reverberił1
}

\begin{abstract}
Background: We aimed to compare the incremental value of contrast myocardial perfusion imaging (MPI) for the detection of intermediate versus severe coronary artery stenosis during dipyridamole-atropine echocardiography (DASE).

Wall motion (WM) assessment during stress-echocardiography demonstrates suboptimal sensitivity to detect coronary artery disease (CAD), particularly in patients with isolated intermediate (50\%-70\%) coronary stenosis.

Methods: We performed DASE with MPI in 150 patients with a suspected chest pain syndrome who were given clinical indication to coronary angiography.

Results and discussion: When CAD was defined as the presence of $\mathrm{a} \geq 50 \%$ stenosis, the addition of MPI increased sensitivity (+30\%) and decreased specificity $(-14 \%)$, with a final increase in total diagnostic accuracy $(+16 \%, p<0.001)$. The addition of MPI data substantially increased the sensitivity to detect patients with isolated intermediate stenosis from $37 \%$ to $98 \%$ ( $p<0.001$ ); the incremental sensitivity was much lower in patients with severe stenosis, from $85 \%$ to $96 \%(p<0.05)$, at the expense of a higher decrease in specificity and a final decrease in total diagnostic accuracy $(-18 \%$, $\mathrm{p}<0.001)$.

Conclusions: The addition of MPI on top of WM analysis during DASE increases the diagnostic sensitivity to detect obstructive CAD, whatever its definition ( $\geq 50 \%$ or $>70 \%$ stenosis), but it is mainly driven by the sensitivity increase in the intermediate group (50\%-70\% stenosis).

The total diagnostic accuracy increased only when defining CAD as $\geq 50 \%$ stenosis, since in patients with severe stenosis ( $>70 \%)$ the decrease in specificity is not counterbalanced by the minor sensitivity increase.
\end{abstract}

\section{Background}

Standard pharmacological stress-echocardiography (SE) has demonstrated suboptimal sensitivity to detect intermediate coronary stenosis, particularly in patients with single-vessel disease, not differently from other techniques based on the detection of inducible wall motion abnormalities [1-3]. Nonetheless the diagnosis of a coronary stenosis between $50 \%$ and $70 \%$ in diameter is relevant to the patient, since it may portend an unfavorable prognosis when associated with reduced coronary flow reserve or fractional flow reserve $[2,4]$.

\footnotetext{
*Correspondence: asqueri@ao.pr.it

${ }^{1}$ Cardiology Division, Azienda Ospedaliero-Universitaria di Parma, Parma, Italy + Contributed equally

Full list of author information is available at the end of the article
}

Both the quantitative measurement of myocardial blood flow reserve and the more practical visual assessment of myocardial perfusion (MPI) during contrast-SE have demonstrated incremental sensitivity on top of wall motion (WM) analysis to diagnose obstructive coronary artery disease (CAD) [5-10].

The study hypothesis is that the potential incremental diagnostic sensitivity and accuracy of MPI during dipyridamole-atropine echocardiography (DASE) is driven by the higher sensitivity in the subset of patients with isolated intermediate coronary stenosis. We performed DASE with the addition of MPI in 150 consecutive patients with a chest pain syndrome who were given clinical indication to elective coronary angiography and we 
analyzed the diagnostic results based on the presence and grade of coronary artery stenosis.

\section{Methods}

\section{Patients}

All patients who were clinically indicated elective coronary angiography between March 2008 and January 2009 for a recent chest pain syndrome were asked their informed consent to be enrolled in the study; the ones who consented underwent DASE within 2 weeks before coronary angiography, in the absence of the following exclusion criteria:

\section{Exclusion criteria}

a) left ventricular ejection fraction (LVEF) $<30 \%$, b) severe valvular disease, c) sustained ventricular arrhythmias or hemodynamic instability, d) insufficient acoustic windows, e) known allergy to sulfonamides.

The study complied with the Declaration of Helsinki. All patients gave written informed consent to the study protocol, which was approved by the Institutional Review Board of our Hospital.

\section{Dipyridamole-atropine echocardiography}

The contrast stress protocol is shown in Figure 1. All images were acquired in the apical 4, 2 and 3 chamber views using S5 broadband transducer (Philips, Eindhoven, Netherlands). Wall motion and myocardial perfusion were assessed at rest and at peak stress. Sonovue (Bracco, Milan, Italy) was infused using a rotating pump (BR-NF100, Bracco, Geneva, Switzerland) at rest and 1 minute after completion of dipyridamole infusion. Low power $(0.10)$ continuous imaging was performed for WM assessment. For MPI image acquisition a flash-replenishment sequence (destructive pulse of 8 frames at a mechanical index of 1.0, followed by low power imaging for 10 cycles) was used both in the continuous (40 frames/sec) and triggered mode (end-systolic at every cardiac cycle) [5].

Care was taken to opacify the left ventricle and the myocardium homogeneously with minimization of attenuation so that it is confined to the atrium prior to acquisition of contrast imaging. To achieve this the infusion rate and gain settings are adjusted (e.g. if the contrast attenuation is seen in the LV cavity, infusion rate is reduced and if the attenuation is optimal, then gains are increased for optimal opacification). Gain settings are considered optimal if the destructive impulses result in complete clearance of microbubbles from the myocardium with very little cavity contrast destruction.

\section{Interpretation of WM and MPI}

Contrast regional WM analysis was evaluated at baseline and at peak stress by using a semi quantitative wall motion score (normal, hypokinesia and akinesia) on a 17segment model of the left ventricle, according to the recommendations of the European Association of Echocardiography [11].

Abnormal WM was defined as the occurrence in at least one segment of either a new dyssynergy in a region with normal rest function or worsening of rest dyssynergy. Resting akinesia becoming dyskinesia or fixed WM abnormalities were not considered a criterion for positivity.

Myocardial perfusion was considered as normal if the myocardium was fully replenished 1.5-2 seconds after the end of flash impulse at peak hyperemia and as abnormal if the myocardium replenishment was delayed beyond 2 seconds. The cut-off for normal replenishment at rest

\section{Stress-echocardiography protocol (High dose Dipyridamole-Atropine)}

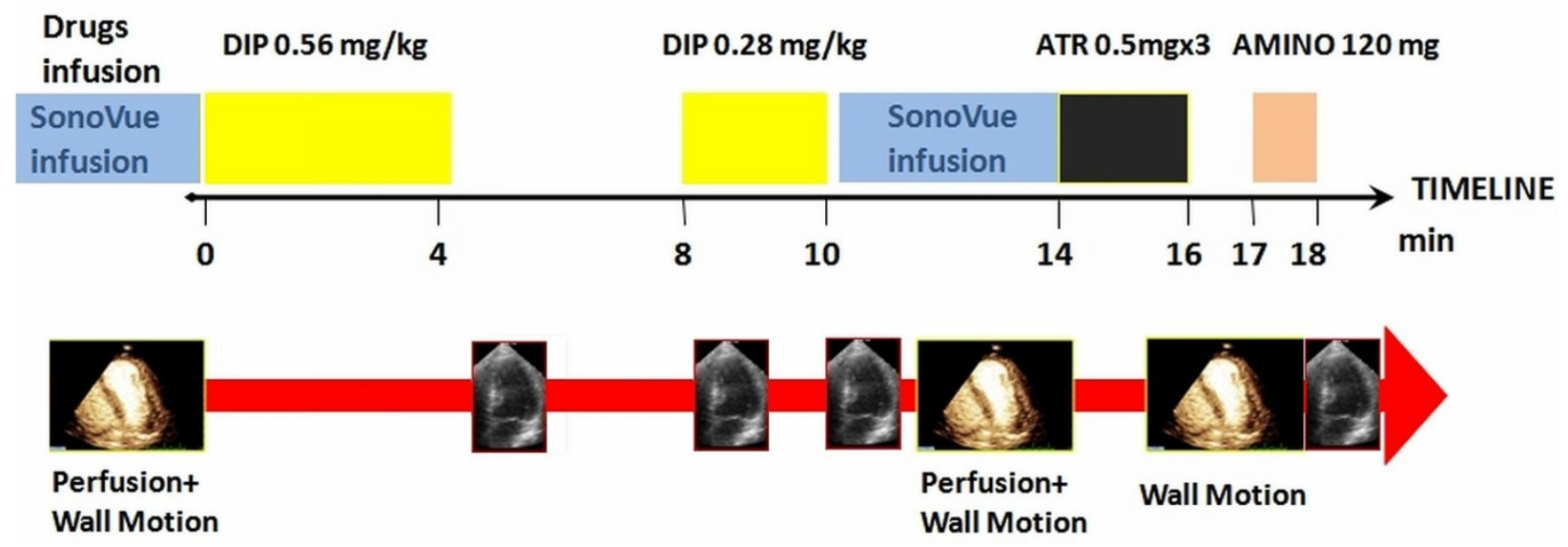

Figure 1 Stress echocardiography protocol. Abbreviations as in table 1. 
was considered 4-5 seconds after the flash impulse. A perfusion defect was scored as fixed or reversible based on its presence or absence at rest. A reversible defect in $\geq 1$ segment was defined as abnormal MPI.

Wall motion and MPI were analyzed separately at 2 different time points by consensus by 2 observers. Disagreement was resolved by a third observer.

\section{Quantitative Coronary Angiography}

Quantitative coronary angiography (QCA) was performed by a cardiologist (F.U.), unaware of the echocardiography results. Any evident stenosis was measured (Qangio XA version 7.0, Medis, Leiden, the Netherlands) and expressed as percent narrowing using the nearest normal-appearing region as the reference. An intermediate stenosis was defined as luminal stenosis in one or more coronary arteries or major branches between $50 \%$ and $70 \%$, while a severe stenosis was defined as $>70 \%$ narrowing.

Patients were divided into subgroups: a) normal coronaries or $<50 \%$ stenosis, b) stenosis between $50 \%$ and $70 \%$ as the worst coronary lesion (INTERMEDIATE group) or c) at least one $>70 \%$ stenosis (SEVERE group). Patients with left main disease $\geq 50 \%$ were also included in the SEVERE group. Multivessel disease was defined when more than one of the three major coronary arteries (or left main trunk) was affected by a $\geq 50 \%$ stenosis. Coronary territories were assigned according to guidelines [11].

\section{Statistical analysis}

Continuous variables were presented as mean and standard deviation and were compared using the Student $t$ test. Categorical variables were examined with a chisquare test when appropriate (expected frequency $>5$ ), otherwise a Fisher exact test was used. Sensitivity, specificity and accuracy were calculated using standard definitions and were presented with 95\% confidence intervals (CI). Differences between sensitivity, specificity and accuracy using WM or WM+MPI were analyzed using $\mathrm{McNe}$ mar's test. A value of $\mathrm{p}<0.05$ (2 sided) was considered significant. Interobserver and intraobserver agreement was determined for both WM and MPI in 30 randomly selected patients. Results were presented as a percentage with a corresponding kappa value. Statistical analysis was performed with SPSS version 15.0 (SPSS Inc, Chicago, Ill).

\section{Results}

\section{Clinical data}

169 consecutive patients met the inclusion criteria; 11 denied informed consent and 8 met exclusion criteria (6 insufficiently echogenic and 2 reporting known allergy to sulfonamides). 150 patients were finally enrolled. Mean age was $70 \pm 9,78(52 \%)$ were males, $115(77 \%)$ had at least 2 traditional risk factors for CAD, 40 (27\%) had diabetes mellitus and 51 (34\%) had a previous myocardial infarction or percutaneous coronary intervention; 47 subjects (31\%) had a final angiographic result of single vessel-disease, 55 (37\%) multivessel, while 48 (32\%) had normal or $<50 \%$ stenosed coronary arteries. All demographic and clinical data were comparable between the INTERMEDIATE and the SEVERE group.

\section{Stress echocardiography}

The prevalence of reversible WM abnormalities was higher in the SEVERE group when compared with the INTERMEDIATE group ( $p<0.001$ ); in contrast, the prevalence of MPI abnormalities was not different between the two groups (Table 1).

Sensitivity, specificity and accuracy for WM and $\mathrm{WM}+\mathrm{MPI}$ in patients with any stenosis $\geq 50 \%$ (INTERMEDIATE+SEVERE groups) or > $70 \%$ only (SEVERE group) are reported in Figure 2, along with sensitivity data for the INTERMEDIATE group.

Sensitivity using WM+MPI resulted significantly higher than using WM data only, whatever the cut-off used to define CAD. The sensitivity increase was highest in the INTERMEDIATE group, from 37\% (CI 25\%-47\%) to $98 \%$ (CI 89\%-100\%), ( $\mathrm{p}<0.001)$.

The sensitivity increase of WM+MPI for CAD $\geq 50 \%$, from $66 \%$ (CI 61\%-70\%) to 96\% (CI 92\%-99\%) (p < 0.001), was only partially counterbalanced by the specificity decrease from 83\% (CI 70\%-92\%) to 69\% (CI 61\%-74\%) (p $<0.05)$; MPI total accuracy resulted higher compared with standalone WM analysis, $87 \%$ (CI 82\%-93) vs $71 \%$ (CI 65\%-78\%), ( $p<0.001)$. On the contrary, in the SEVERE group the decrease in specificity obtained with the addition of MPI data was much higher, from $74 \%$ (CI $67 \%-79 \%$ ) to $38 \%$ (CI 29\%-49\%, p < 0.001), than the sensitivity increase, from $85 \%$ (CI 76\%-91\%) to 96\% (CI 92\%$99 \%), \mathrm{p}<0.05)$, with final lower total accuracy, from $79 \%$ (CI 72\%-83\%) to 61\% (54-65) $(\mathrm{p}<0.001)$.

The addition of MPI identified 25 more true positive patients than WM in the INTERMEDIATE group; among them, in 13 patients (52\%) the intermediate stenosis detected was in the anterior circulation (anterior descending coronary artery), in 10 patients (40\%) in the posterior circulation (right or circumflex coronary artery) and in 2 patients (8\%) both in the anterior and posterior circulation.

The addition of MPI identified 6 more true positive patients than WM in the SEVERE group, 5 with stenosis both in the anterior and posterior circulation (multivessel disease) and 1 with a single stenosis in the anterior circulation (single-vessel disease).

Figure 3 shows the average stenosis diameter in patients who underwent QCA, classified into three subgroups 
Table 1: Clinical characteristics and echocardiographic data in the 3 angiographic categories, according to QCA results.

\begin{tabular}{|c|c|c|c|c|}
\hline Study group $n=150$ & No CAD & CAD 50\%-70\% & CAD $>70 \%$ & p value \\
\hline Patients n (\%*) & 48 & 41 & 61 & - \\
\hline Age, mean $( \pm 1 S D), y$ & $64(10)$ & $69(9)$ & $70(9)$ & ns \\
\hline Men, n (\%) & $26(54)$ & $24(59)$ & $40(65)$ & ns \\
\hline Risk factors $\geq 2, \mathrm{n}(\%)$ & $37(77)$ & $34(85)$ & $51(84)$ & ns \\
\hline Hypertension, n (\%) & $32(67)$ & $30(73)$ & $42(69)$ & $\mathrm{ns}$ \\
\hline Diabetes Mellitus n (\%) & $6(13)$ & $12(29)$ & $19(31)$ & ns \\
\hline Prior myocardial infarction or $\mathrm{PCl} n$ (\%) & $11(23)$ & $13(32)$ & $23(38)$ & ns \\
\hline Presence of baseline WM abnormalities, $\mathrm{n} \%$ & $10(21)$ & $14(34)$ & $24(39)$ & ns \\
\hline Baseline LVEF, \% & $57 \pm 8$ & $54 \pm 9$ & $52 \pm 10$ & ns \\
\hline Abnormal WM, n (\%) & $8(17)$ & $15(37)$ & $52(85)$ & $p<0.001$ \\
\hline Abnormal myocardial perfusion $\mathrm{n}(\%)$ & $15(31)$ & $40(98)$ & $58(95)$ & ns \\
\hline Peak RPP mean $( \pm 1 \mathrm{SD})$ & $15124(3140)$ & 15416(3684) & $14615(2705)$ & ns \\
\hline
\end{tabular}

based on SE results: MPI-/WM-, MPI+/WM-, MPI+/ $\mathrm{WM}+$.

Figure 4 shows a typical DASE examination resulting positive for MPI after dipyridamole with normal peak WM, in a patient finally diagnosed with two-vessel $50 \%$ $70 \%$ coronary artery stenosis (see also Additional File 1).

\section{Intra/interobserver variability}

The intraobserver agreement on the presence or absence of reversible visual MPI or WM abnormality after dipyridamole is respectively 97\% (Kappa $=0.91$ ) and 100\% (Kappa $=1)$; the interobserver agreement data is respectively 93\% $($ Kappa $=0.83)$ and 90\% $($ Kappa $=0.73)$.

\section{Feasibility and Safety}

The test could be performed and interpreted both for WM and MPI in all enrolled patients, after the initial exclusion of 8 patients for poor acoustic windows or sulfonamide allergy. Feasibility of DASE was consequently $95 \%$.
After dipyridamole infusion mild flushing or headache were commonly reported. There were no serious or irreversible adverse events. There was no suggestion for any direct adverse effect related to contrast infusion.

\section{Discussion}

Potential clinical value of detecting intermediate stenosis An intermediate stenosis has a negative prognostic impact on future cardiac events only if associated with a reduced coronary (CFR) or fractional flow reserve (FFR); CFR or FFR have been found reduced in more than half of patients with known intermediate stenosis, non-invasively studied using stress-echocardiography (CFR assessment), or invasively studied with Doppler flow-wire measurements (FFR assessment) [2,4]. The detection of an intermediate stenosis may consequently be relevant for the clinical prognosis of patients, although the management of such intermediate stenosis needs to be tailored on a single patient basis. 


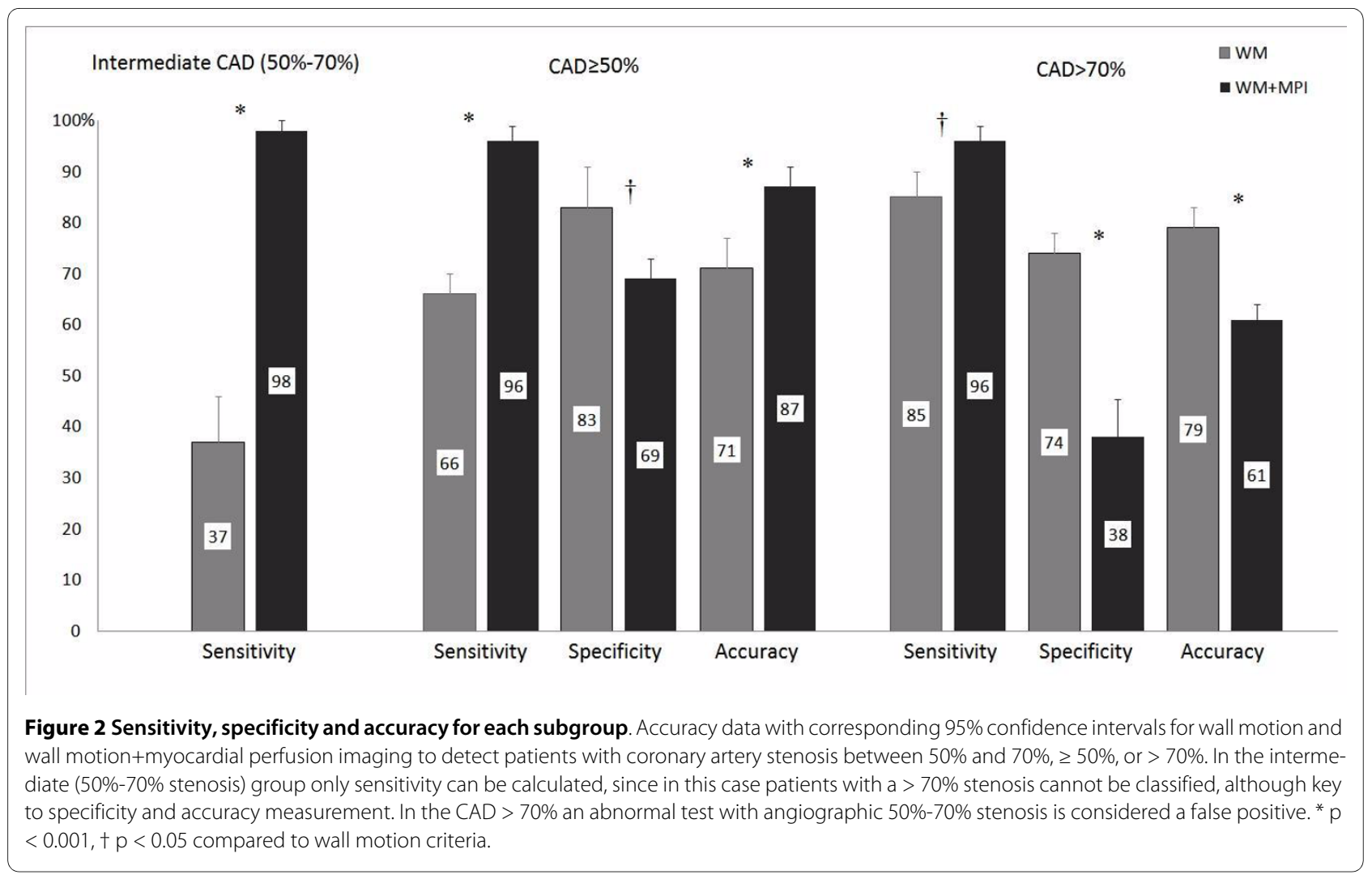

The long-term prognostic value of MPI for hard cardiac events, independently by WM, has been clearly demonstrated [12,13], however no single study specifically addressed the prognostic value of a MPI defect subtended by an intermediate stenosis.

In conclusion, whether a reversible perfusion defect in patients with isolated intermediate stenosis implies an unfavorable prognosis is a question that remains unanswered.

\section{Wall motion criteria in intermediate stenosis}

Contemporary studies using either dipyridamole or dobutamine echocardiography clearly demonstrated the suboptimal sensitivity of pharmacologic stress protocols to detect intermediate stenosis when using standard WM criteria $[1,2,14,15]$. In a study using MPI in conjunction with dobutamine-atropine SE, pooled sensitivity of WM criteria for intermediate stenosis was only $30 \%$, increasing to $67 \%$ when MPI was added [1].

A recent study, in which high dose dipyridamole was used, reported a $20 \%$ sensitivity for WM criteria to detect $50 \%-75 \%$ coronary stenosis in patients with isolated intermediate stenosis of the anterior descending coronary artery; transthoracic Doppler CFR in this study was very effective to overcome the limited sensitivity of WM, but in the clinical practice the undisputable value of non- invasive CFR measurement is technically limited to the anterior coronary circulation [2].

The incremental value of contrast MPI is instead maintained for all coronary territories, as previously reported [1]. In our study the anterior circulation was affected in only $60 \%$ of patients with isolated intermediate stenosis.

Another study demonstrated a major step-up in sensitivity for detection of an intermediate stenosis (associated with fractional flow reserve $<0.75$ ) by the use of contrast for left ventricle opacification during dobutamine echocardiography; in this case sensitivity increased from $48 \%$ for standard echocardiography without contrast, to $83 \%$ with the use of contrast for opacification, reflecting the usefulness of better endocardial border delineation [15].

Sensitivity data in this study, which is otherwise particularly interesting for the choice of a hemodynamic functional endpoint, are presumably much higher than real because of the bias of performing and interpreting the tests in patients with previously known intermediate stenosis, which was the reason for stress-testing.

\section{Clinical value of contrast MPI, costs and safety issues}

Contrast use to date is not approved specifically for MPI by the European Medicines Agency (EMEA) and its use remains consequently investigational. In our study contrast MPI is useful to increase the sensitivity of pharma- 


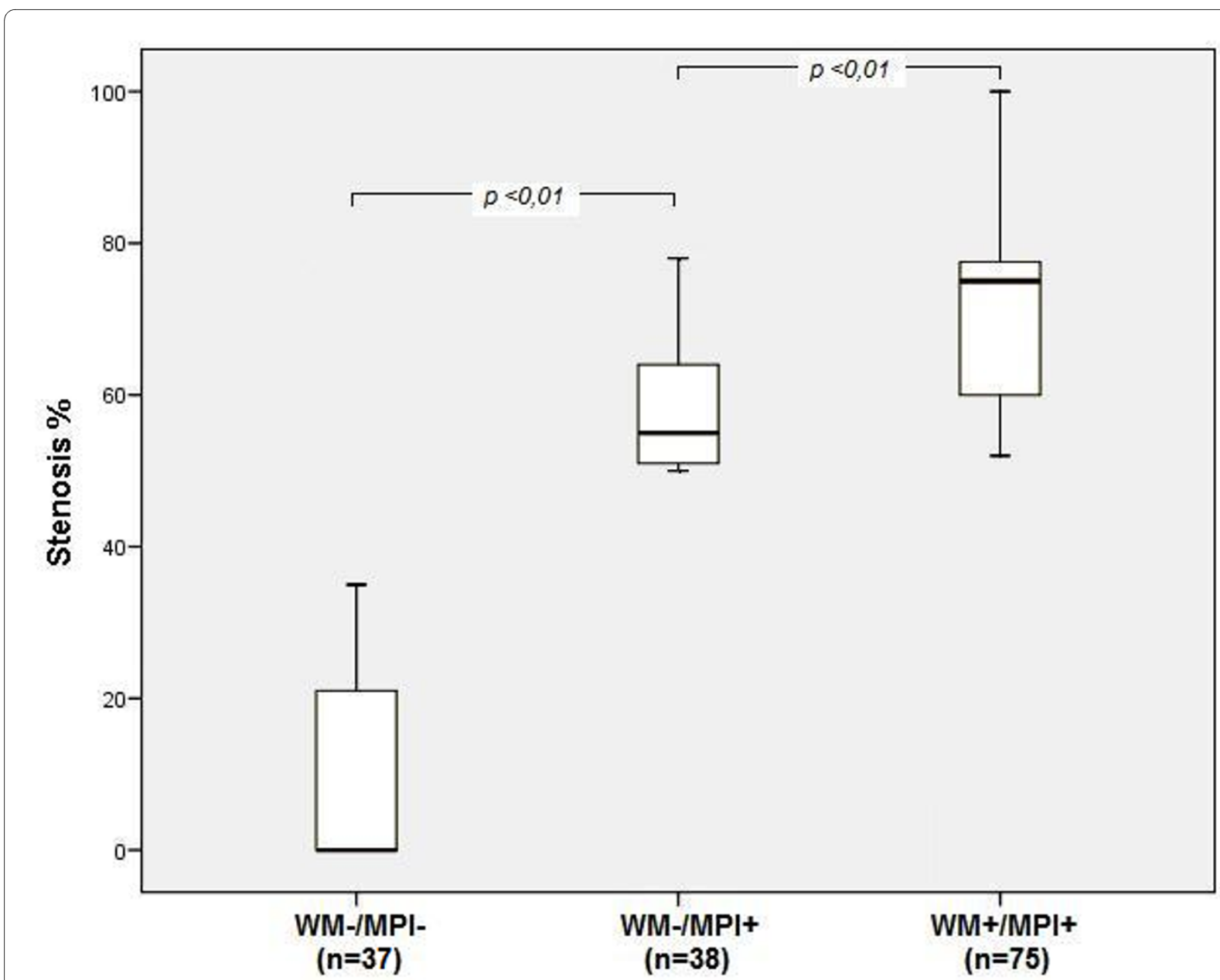

Figure 3 Average stenosis diameter. Average stenosis diameter in patients who underwent QCA, classified into three subgroups based on SE results: MPI-MM-, MPI+/WM-, MPI+/WM+. Abbreviations as defined in table 1.

cologic stress-echocardiography when the diagnosis of less severe $\mathrm{CAD} /$ stenosis is the diagnostic endpoint, while for more severe CAD/stenosis the profound loss in specificity leads to a significant loss in accuracy, compared with standard WM analysis; whether the diagnostic cost-benefit profile of adding contrast MPI is worth the potential safety issues and contrast-related costs is still a matter of discussion. An isolated MPI abnormality (ie, with normal WM) should not be used as the sole indication to coronary angiography, due to its low specificity for epicardial obstructive CAD.

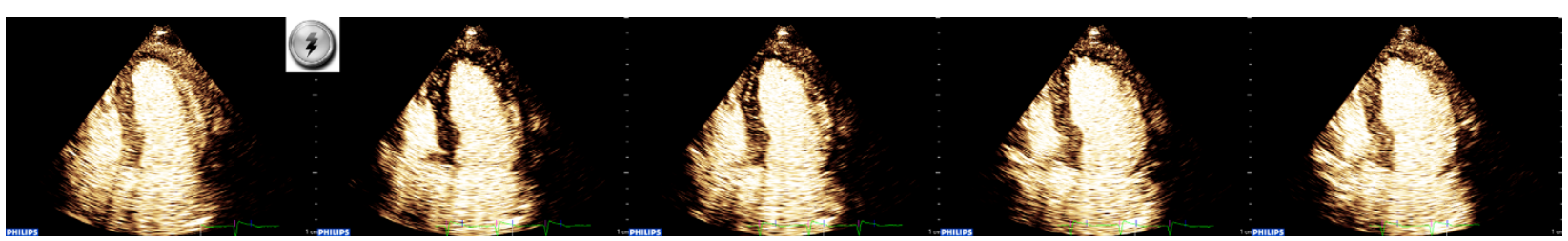

Figure 4 Flash replenishment sequence. Assessment of myocardial perfusion after dipyridamole. From left to right: uniform transmural perfusion seen in apical 4-chamber before flash, then images taken 1, 3, 4 and 8 cardiac cycles after microbubbles destruction. Perfusion defects become apparent after flashing (flash icon in the figure) both in the anterior descending and circumflex coronary arteries perfusion territories, still detectable after $8 \mathrm{cycles}$; the patient had no clear WM abnormality, even if mild tardokinesia of the septum was suspected. Angiography confirmed obstructive twovessel disease with both stenosis ranging between $50 \%$ and $70 \%$. Abbreviations as defined in table 1. 


\section{False positives and coronary angiography}

Patients with a positive provocative test (whatever stresstest considered) and no obstructive epicardial CAD at coronary angiography are usually defined as false-positives. It is now widely recognized that in many different clinical situations (microvascular disease, diffused coronary atherosclerosis, subcritical disease with coronary remodeling, myocardial bridges, coronary spasm, cardiomyopathies and others) coronary blood flow under stress condition may be significantly reduced in the absence of epicardial $>50 \%$ stenosis, up to the level capable to cause chest pain, ECG and perfusion abnormalities and, less frequently, WM abnormalities [16]. MPI is intrinsically incline to a higher "false positivity" rate than WM, due to its earlier role in the pathophysiology of the ischemic cascade.

Few stress-echocardiography studies reported that patients with so called "normal coronaries" and an abnormal stress-test (for WM or CFR-LAD) have a worse cardiac prognosis than patients with a normal test $[17,18]$.

\section{Limitations}

The specificity of WM in our study was lower than reported by several previous studies; this may be due to the non-conservative reading criteria adopted $(\geq 1$ segment with new dyssynergy in a region with normal rest function or worsening of rest dyssynergy), that were anyway similarly applied to MPI ( $\geq 1$ segment with reversible MPI defect). Analysis of echocardiographic data was performed on a patient-basis and not on a territory-basis, since we felt it inappropriate, stress-echocardiograms being always interrupted in our centre at the very first sign of positivity, for safety reasons. The limitations of angiographic comparisons in relation to the discrepancy between stenosis severity and functional significance are pertinent to our study too. Use of angiographic data alone ignores patients with non-ischemic cardiomyopathies, the previous occurrence of coronary events in patients without significant stenosis or microcirculation disease. Wall motion abnormalities or MPI defects in these patients are classified as false-positives; in our experience this has a major impact on MPI data, more than on WM data.

\section{Conclusion}

The current study shows that most of the incremental diagnostic sensitivity of MPI over WM analysis during DASE originates from patients with coronary artery stenosis of intermediate severity, defined as $50 \%-70 \%$ in luminal diameter. Interestingly, MPI maintains incremental sensitivity in the SEVERE group also, but clinically less significant. In the SEVERE group, however, the addition of MPI decreased the specificity more than how it increased the sensitivity, resulting in lower total accuracy, compared with WM assessment. The opposite is true when targeting coronary artery stenosis $\geq 50 \%$.

We conclude that MPI should be used with caution if the endpoint of the test is the identification of angiographically severe (> $70 \%$, so called "critical") stenosis. In this case the trade-off of MPI between sensitivity and specificity becomes unfavorable for MPI, while standalone WM assessment (using left ventricle contrast opacification, as we did in our study) maintains a higher total accuracy.

\section{Additional material}

Additional file 1 Abnormal MPI after dipyridamole with normal peak WM in a patient finally diagnosed with two-vessel 50\%-70\% coronary artery stenosis.

\section{Abbreviations}

WM: wall motion; CAD: coronary artery disease; MPI: contrast myocardial perfusion imaging; DASE: dipyridamole-atropine stress echocardiography; QCA: quantitative coronary angiography; LVEF: left ventricular ejection fraction; CFR: coronary flow reserve; FFR: fractional flow reserve; RPP: rate-pressure product

\section{Competing interests}

The authors declare that they have no competing interests.

\section{Authors' contributions}

NG performed the echostress and drafted the manuscript, FR analyzed the CFR data, AS drafted the manuscript and performed the statistical analysis, FU made QCA analysis, CR drafted the manuscript. All authors read and approved the final manuscript

\section{Author Details}

${ }^{1}$ Cardiology Division, Azienda Ospedaliero-Universitaria di Parma, Parma, Italy and ${ }^{2}$ Cardiology Division, Umberto I $^{\circ}$ Hospital, Mestre-Venice, Italy

Received: 30 March 2010 Accepted: 6 May 2010

Published: 6 May 2010

\section{References}

1. Elhendy A, O'Leary EL, Xie F, McGrain AC, Anderson JR, Porter TR: Comparative accuracy of real-time myocardial contrast perfusion imaging and wall motion analysis during dobutamine stress echocardiography for the diagnosis of coronary artery disease. J Am Coll Cardiol 2004, 44:2185-91

2. Rigo F, Sicari R, Gherardi S, Djordjevic-Dikic A, Cortigiani L, Picano E: Prognostic value of coronary flow reserve in medically treated patients with left anterior descending coronary disease with stenosis $51 \%$ to 75\% in diameter. Am J Cardio/ 2007, 100:1527-31.

3. Nagel E, Lehmkuhl HB, Bocksch W, Klein C, Vogel U, Frantz E, Ellmer A, Dreysse $\mathrm{S}$, Fleck E: Noninvasive diagnosis of ischemia-induced wall motion abnormalities with the use of high-dose dobutamine stress MRI: comparison with dobutamine stress echocardiography. Circulation 1999, 99:763-70.

4. Berger A, Botman KJ, MacCarthy PA, Wijns W, Bartunek J, Heyndrickx GR Pijls NH, De Bruyne B: Long-term clinical outcome after fractional flow reserve-guided percutaneous coronary intervention in patients with multivessel disease. J Am Coll Cardiol 2005, 46:438-42.

5. Wei K, Ragosta M, Thorpe J, Coggins M, Moos S, Kaul S: Noninvasive quantification of coronary blood flow reserve in humans using myocardial contrast echocardiography. Circulation 2001, 103:2560-5.

6. Moir S, Haluska BA, Jenkins C, Fathi R, Marwick TH: Incremental benefit of myocardial contrast to combined dipyridamole-exercise stress echocardiography for the assessment of coronary artery disease. Circulation 2004, 110:1108-13.

7. Gaibazzi N, Reverberi C, Squeri A, De laco G, Ardissino D, Gherli T: Contrast stress echocardiography for the diagnosis of coronary artery disease in 
patients with chest pain but without acute coronary syndrome: incremental value of myocardial perfusion. J Am Soc Echocardiogr 2009, 22(4):404-10.

8. Lipiec P, Wejner-Mik P, Krzeminńska-Pakuła M, Kuśnierek J, Płachcińska A, Szumiński R, Peruga JZ, Kasprzak JD: Accelerated Stress Real-time Myocardial Contrast Echocardiography for the Detection of Coronary Artery Disease: Comparison with 99mTc Single Photon Emission Computed Tomography. J Am Soc Echocardiogr 2008, 21:941-7.

9. Peltier M, Vancraeynest D, Pasquet A, Ay T, Roelants V, D'hondt AM, Melin $J A$, Vanoverschelde JL: Assessment of the physiologic significance of coronary disease with dipyridamole real-time myocardial contrast echocardiography. Comparison with technetium-99 m sestamibi single-photon emission computed tomography and quantitative coronary angiography. J Am Coll Cardiol 2004, 43:257-64

10. Jeetley P, Hickman M, Kamp O, Lang RM, Thomas JD, Vannan MA, Vanoverschelde JL, Wouw PA van der, Senior R: Myocardial contrast echocardiography for the detection of coronary artery stenosis: a prospective multicenter study in comparison with single-photon emission computed tomography. J Am Coll Cardiol 2006, 47:141-5.

11. Sicari R, Nihoyannopoulos P, Evangelista A, Kasprzak J, Lancellotti P, Poldermans D, Voigt JU, Zamorano JL, European Association of Echocardiography: Stress echocardiography expert consensus statement: European Association of Echocardiography (EAE) (a registered branch of the ESC). Eur J Echocardiogr 2008, 9:415-37.

12. Tsutsui JM, Elhendy A, Anderson JR, Xie F, McGrain AC, Porter TR: Prognostic value of dobutamine stress myocardial contrast perfusion echocardiography. Circulation 2005, 112:1444-50.

13. Dawson D, Kaul S, Peters D, Rinkevich D, Schnell G, Belcik JT, Wei K: Prognostic value of dipyridamole stress myocardial contrast echocardiography: comparison with single photon emission computed tomography. J Am Soc Echocardiogr 2009, 22:954-60.

14. Tsutsui JM, Elhendy A, Xie F, O'Leary EL, McGrain AC, Porter TR: Safety of Dobutamine Stress Real-Time Myocardial Contrast Echocardiography. J Am Coll Cardiol 2005, 45:1235-42.

15. Jung PH, Rieber J, Störk S, Hoyer C, Erhardt I, Nowotny A, Voelker W, Weidemann F, Ertl G, Klauss V, Angermann CE: Effect of contrast application on interpretability and diagnostic value of dobutamine stress echocardiography in patients with intermediate coronary lesions: comparison with myocardial fractional flow reserve. Eur Heart J 2008, 29:2536-43.

16. Gaibazzi N, Reverberi C: False-positive stress tests... or false-negative rest angiograms? J Am Coll Cardiol 2009, 54:e9.

17. Sicari R, Rigo F, Cortigiani L, Gherardi S, Galderisi M, Picano E: Additive prognostic value of coronary flow reserve in patients with chest pain syndrome and normal or near-normal coronary arteries. Am J Cardiol 2009, 103:626-31.

18. Sicari R, Palinkas A, Pasanisi EG, Venneri L, Picano E: Long-term survival of patients with chest pain syndrome and angiographically normal or near-normal coronary arteries: the additional prognostic value of dipyridamole echocardiography test (DET). Eur Heart J 2005, 26:2136-41

doi: $10.1186 / 1476-7120-8-16$

Cite this article as: Gaibazzi et al., Incremental value of contrast myocardial perfusion to detect intermediate versus severe coronary artery stenosis during stress-echocardiography Cardiovascular Ultrasound 2010, 8:16

\section{Submit your next manuscript to BioMed Central} and take full advantage of:

- Convenient online submission

- Thorough peer review

- No space constraints or color figure charges

- Immediate publication on acceptance

- Inclusion in PubMed, CAS, Scopus and Google Scholar

- Research which is freely available for redistribution

Submit your manuscript at www.biomedcentral.com/submit
C Biomed Central 